\title{
IL-33/ST2 axis mediates hyperplasia of intrarenal urothelium in obstructive renal injury
}

Wei-Yu Chen $\mathbb{B}^{1}$, Jenq-Lin Yang ${ }^{1}$, Yi-Hsiu Wu ${ }^{2}$, Lung-Chih $\mathrm{Li}^{1,3}$, Ru-Fang Li ${ }^{1}$, Ya-Ting Chang ${ }^{1}$, Lo-Hsin Dai ${ }^{1}$, Wan-Chen Wang ${ }^{1}$ and Ya-Jen Chang ${ }^{2}$

\begin{abstract}
The monolayered intrarenal urothelium covers the renal papilla and ureteropelvic junction (UPJ). In response to increased renal pressure during obstruction or ischemic injuries, intrarenal urothelial cells begin to proliferate and form a multilayered urothelium. Little is known regarding the mechanism and pathophysiological role of urothelium hyperplasia during renal obstruction. In this study, we investigated the expression of interleukin (IL)-33, an IL-1 family cytokine, in kidneys with unilateral ureteral obstruction (UUO)-induced obstructive injury. IL-33 levels in hydronephrotic urine and serum were upregulated 2 days after UUO. The number of ST2-expressing immune cells was increased in the UUO kidney. We found that IL-33 was upregulated in vimentin-positive cells in the cortical and medullar layers and the UPJ stroma. Moreover, IL-33 expression was predominantly induced in multilayered keratin 5positive urothelial cells in the UPJ. IL-33 was not detected in terminally differentiated superficial umbrella cells expressing uroplakin 3a. In vivo, we confirmed that deficiency of IL33 or its receptor ST2 attenuated UUO-induced hyperplasia of the UPJ urothelium. Deficiency of IL33 attenuated the expression of UUO-induced type 2 inflammatory cytokines and upregulated uroplakins and urothelial differentiation signaling in UPJ tissues. Our results collectively suggest that the IL-33/ST2 axis mediates the activation of innate immune responses and contributes to urothelial hyperplasia by regulating urothelial differentiation in obstructive kidney injury.
\end{abstract}

\section{Introduction}

Interleukin (IL)-33 is an IL-1 family cytokine that is crucial in mediating both innate and adaptive immune responses as a nuclear alarmin ${ }^{1}$. IL-33 elicits immune responses by activating various types of immune cells that express the IL-33 receptor ST2, including mast cells, eosinophils, neutrophils, helper $2 \mathrm{~T}$ cells, and most importantly, group 2 innate lymphoid cells (ILC2s), which secrete large amounts of IL-5 and IL-13 ${ }^{2-4}$. Recently, prolonged activation of ILC2s by IL-33 was shown to

Correspondence: W-Y. Chen (wychen624@cgmh.org.tw) or Y-J. Chang (yajchang@ibms.sinica.edu.tw)

${ }^{1}$ Institute for Translational Research in Biomedicine, Kaohsiung Chang Gung Memorial Hospital, Kaohsiung, Taiwan

${ }^{2}$ Institute of Biomedical Sciences, Academia Sinica, Taipei, Taiwan

Full list of author information is available at the end of the article protect mice from adriamycin-induced glomerulosclerosis $^{5}$. However, proinflammatory roles of IL-33 in renal injury have also been demonstrated in cisplatininduced acute kidney injury ${ }^{6}$. Our previous study demonstrated that deficiency of IL-33 reduces renal fibrosis and tubular cell loss in the unilateral ureteral obstruction (UUO) model $^{7}$. In the context of different renal injury models, IL-33 likely functions as a doubleedged sword upon tissue injury: on the one hand, IL-33 is critical for tissue repair or eliminating infection, while on the other, excessive production of IL-33 can cause tissue and organ damage ${ }^{8,9}$.

Obstructive nephropathy is the major etiology of chronic renal failure during infancy and childhood ${ }^{10,11}$. Obstructive nephropathy leads to the formation of atubular glomeruli, proximal tubular cell loss, immune cell 
infiltration, collecting duct remodeling, and interstitial fibrosis ${ }^{12}$. The UUO model is widely used to study the mechanisms of tubulointerstitial fibrosis via surgically induced obstructive renal injury ${ }^{13}$. In addition to the remodeling of the renal tissues, intrarenal urothelium hyperplasia was observed in experimental hydronephrosis and pyelonephritis ${ }^{14,15}$. Proliferation of the intrarenal urothelium is also observed in mouse models of ischemic renal injury ${ }^{16}$ and kidney obstruction ${ }^{17}$ and in rats with calcium oxalate stones ${ }^{18}$. Recent studies have also revealed that ischemia- and renal obstruction-induced intrarenal urothelial cell proliferation involves activation of the fibroblast growth factor 7 (FGF-7) and FGF receptor 2 (FGFR2) pathway ${ }^{16,17}$. However, little is known regarding the regulation and role of urothelium hyperplasia in obstructive uropathy. In humans, urothelial hyperplasia in children with congenital ureteropelvic junction (UPJ) obstruction is associated with type 2 inflammatory cytokines ${ }^{19}$. We therefore hypothesized that an IL-33-mediated type 2 immune response might contribute to the pathogenesis of urothelial hyperplasia in obstructive nephropathy. In this study, we investigated the role of the IL-33/ST2 axis in UUO-induced obstructive nephropathy, and we identified a previously unrecognized function of IL-33 in mediating intrarenal urothelium hyperplasia.

\section{Materials and methods Animal models}

All animal experiments were conducted in accordance with the Guide for the Use and Care of Laboratory Animals and approved by the IACUC Committee of Kaohsiung Chang Gung Memorial Hospital, Kaohsiung, Taiwan. IL33knockout (IL33KO) and ST2-knockout (ST2KO) mice were maintained in the C57BL/6 background as previously described $^{7,20}$. The primer sequences for mouse genotyping are listed in Supplementary Table S1.

Eight-week-old male wild-type C57BL/6, IL33KO, and ST2KO mice were generally anesthetized using isoflurane (2\%) and then subjected to unilateral ureteral ligation by ligating the left distal ureter with a $4 / 0$ silk suture through a low midline abdominal incision ${ }^{12,13}$. Mice were sacrificed on $2,4,7,10$, and 14 days after UUO surgery. Nonobstructed and obstructed kidney samples were collected and divided into two equal sections for paraffin sectioning. Hydronephrotic urine samples were collected from the obstructed kidneys using $27 \mathrm{G}$ needles. Control urine was collected from the bladder. UPJ tissues from control and obstructed kidneys were further dissected and collected for RNA isolation and protein lysate preparation.

\section{Flow cytometry}

Kidneys were minced and then digested with collagenase $\mathrm{B}$ (Worthington) for $30 \mathrm{~min}$ at $37^{\circ} \mathrm{C}$ with rotation, and the homogenate was sheared by 18 -gauge needles in the midst of enzymatic digestion. Next, the homogenate was strained through $100-\mu \mathrm{m}$ mesh, and then the residual cells were pelleted and subjected to ACK buffer (Gibco) treatment before final suspension in FACS buffer. For surface staining of myeloid cells, single-cell suspensions were first stained with Fixable Viability Dye (eBioscience). Fc receptors were blocked with 2.4G2 conditional medium before antibody staining. Among $\mathrm{CD} 45+$ leukocytes, macrophages $(\mathrm{F} 4 / 80+)$, $\mathrm{T}$ cells or ILCs (Thy1.2+), neutrophils (Ly6G+Ly6C+), and monocytes (Ly6C+MHCII-) can be identified ${ }^{21}$. We defined lineage-negative (Lin-, including $\mathrm{CD} 3 \varepsilon, \mathrm{CD} 11 \mathrm{~b}$, CD11c, CD19, CD49b, F4/80, FceRI) but Thy1.2+ cells as ILCs and double-positive cells as T cells during surface staining. Additionally, a Foxp3 Staining Buffer Set (eBioscience) and anti-mouse GATA3 antibody were used to identify ILC2s (Lineage-Thy1.2+GATA3+). After staining, the cells were washed and re-suspended in FACS buffer and subjected to flow cytometry. Data were analyzed by LSRII (BD Biosciences) and by FlowJo v. 10.1 (TreeStar). A list of antibodies used in flow cytometry is provided in Supplementary Table S2. The gating strategies for flow cytometry analyses for myeloid cells and ILCs in the kidney samples are shown in Supplementary Figure S1.

Immunofluorescent staining and histological assessments

Mouse kidneys were fixed with $4 \%$ paraformaldehyde, embedded in paraffin, sectioned, and stained with standard immunohistochemistry and fluorescent microscopy methods as previously described ${ }^{20,7}$. An additional antigen retrieval step was applied in all experiments by heating samples in a Tris-based buffer ( $\mathrm{pH} 9.0)$ to $95^{\circ} \mathrm{C}$ for $20 \mathrm{~min}$. Primary antibodies are listed in Supplementary Table S2. Alexa Fluor-conjugated secondary antibodies were purchased from ThermoFisher. Slides were incubated with 1\% Sudan black (Sigma-Aldrich) in 75\% ethanol at room temperature for $20 \mathrm{~min}$ to reduce tissue auto-fluorescence before mounting. Confocal images were captured using an Olympus FLUOVIEW FV10i confocal microscope. Scanning of the immunofluorescent images of the whole kidney tissue was performed using TissueFAXS (TissueGnostics). For quantification of urothelium thickness, paraffin sections were stained with haematoxylin and eosin. Bright-field images were scanned using 3D Histech Pannoramic MIDI (3DHISTECH). The average urothelium thickness of the UPJ was calculated using Pannoramic viewer (3DHISTECH).

\section{Quantitative RT-PCR}

For quantitative RT-PCR (qRT-PCR) analysis of gene expression, UPJ tissues from C57BL/6 or IL33-knockout (IL33KO) mice with UUO $(n=5)$ were harvested on day 4 
after UUO surgery (Supplementary Figure S2). Total RNA was isolated using TRIzol reagent (Invitrogen). cDNA was synthesized from $1 \mu \mathrm{g}$ of total RNA and random hexamers using the TaqMan Reverse Transcription kit (Applied Biosystems). qRT-PCR was performed using an ABI 7300 Real-Time PCR system (Applied Biosystems). The primer sequences used are listed in Supplementary Table S3. The UPJ tissue expression levels of genes related to a type 2 immune response or urothelial differentiation and those of uroplakins did not show a significant difference between wild-type and IL33KO mice. Therefore, we performed a two-dimensional principal component analysis (PCA) to simplify the qRT-PCR results. The first component (PC1) is defined as the $\log 2$ value of the fold change in gene expression in UUO UPJ tissues versus non-obstructed control tissues from wild-type C57BL/6 mice and represents UUO-mediated gene regulation. The second component (PC2) is defined as the log2 value of the fold change in gene expression in IL33KO UUO UPJ tissues versus C57BL/6 UUO UPJ tissues and represents IL33-dependent gene regulation in the UUO UPJ tissues. Heatmaps were generated using the online tool GENE-E (https://software. broadinstitute.org/GENE-E/, Broad Institute).

\section{Western blot and ELISA}

Harvested UPJ tissues were lysed using RIPA buffer (Sigma) according to the manufacturer's instructions. The primary antibodies anti-SHH (AB135240, Abcam) and anti-UPK3a (AB187646, Abcam) were used to identify target proteins. A secondary horseradish peroxidaseconjugated antibody was used at a 1:5000 dilution (Millipore). The ECL advanced system (GE Healthcare) was used to detect target proteins. Mouse serum or urine levels of IL-33 (DY413, R\&D System), IL-5 (DY405, R\&D System), IL-13 (DY413, R\&D System), SHH (DY461, R\&D System), BMP4 (EK0316, Boster), and BMP5 (LS-F20454, LSBio) were analyzed using enzyme-linked immunosorbent assay (ELISA) kits according to the manufacturers' instructions.

\section{Statistical analyses}

Data are presented as means \pm SEM. For experiments in which differences between two groups were evaluated, data were analyzed using non-parametric Mann-Whitney $U$-tests.

\section{Results}

\section{UUO upregulated IL-33 urine and serum levels}

To determine the expression of IL-33 in kidney tissues and the UPJ urothelium, we performed UUO surgery to induce UUO in C57BL/6 mice. Pre-surgery urine samples were collected from the bladder as a control. Hydronephrotic urine samples were collected from obstructed kidneys for ELISA analyses of IL-33 (Fig. 1a), IL-5 (Fig. 1b), and IL-13 (Fig. 1c). Urine levels of IL-33, IL-5, and IL-13 in the obstructed kidney were elevated at 4 days post-UUO. Serum levels of IL-33 (Fig. 1d), IL-5 (Fig. 1e), and IL-13 (Fig. 1f) were upregulated on day 2 post-UUO and then declined to basal levels, suggesting that circulating IL-33 was upregulated by an acute inflammatory response to the early insult of kidney obstruction.

\section{Infiltration and expansion of immune cells and ST2+ leukocytes in UUO kidneys}

As the peak IL-33 levels were observed in the urine from obstructed kidneys 4 days post-UUO, we sought to identify changes in immune and non-immune cells in the kidneys following UUO. We found that the total number of CD45+ leukocytes increased dramatically, whereas the number of CD45- non-leukocytes decreased in the UUO kidney (Fig. 2a). Among CD45+ leukocytes, macrophages and Thy1.2+ cells ( $T$ cells and ILCs) comprised the major populations of immune infiltrate, and numbers of macrophages, T cells, ILCs, neutrophils, monocytes, and other un-characterized immune cells were elevated in the UUO kidney (Fig. 2b and Supplementary Figure S1). The total ILC population (CD45+Lin-Thy1.2+) and the ILC2 population (CD45+Lin-Thy1.2+GATA3+), a crucial target of IL-33, were also increased in the UUO kidney (Fig. 2c). Among the increased immune infiltrates in the UUO kidney, macrophages comprised $46.5 \%$ of total CD45+ leukocytes (Fig. 2d). We next analyzed the expression of the IL-33 receptor ST2 among leukocytes and non-leukocytes. Interestingly, although the total number of CD45- non-leukocytes was reduced in the UUO kidney, ST2+CD45- cells were elevated in the UUO kidney. Numbers of ST2+ macrophages, T/ILCs, neutrophils, and monocytes were elevated in UUO kidneys compared with those in non-obstructed control kidneys (Fig. 2e). Among the ST2+ cells, CD45- nonleukocytes, neutrophils, macrophages, T/ILCs, and monocytes comprised 34.8\%, 22.3\%, 23.1\%, 16.2\%, and $3.6 \%$, respectively (Fig. 2f). UUO induced a 30 -fold increase in the number and 4.6-fold increase in the percentage of ST2+ neutrophils in UUO kidneys compared with those in non-obstructed kidneys (Fig. 2e, f). These results together indicate that there is an increased infiltration and expansion of ST2 + innate immune cells in the kidney following obstructive injury.

\section{UUO upregulated IL-33 expression in vimentin+ tubulointerstitial cells}

We next examined the source of IL-33 in the kidney. On day 4 post-UUO surgery, the dilation of the renal pelvic space was observed in the obstructed kidney (Fig. 3a). Immunofluorescent staining revealed increased IL-33 expression in the cortex, medulla, and UPJ urothelium. IL-33-positive staining co-localized with vimentin+ interstitial cells in the cortical and medullar layers in the 


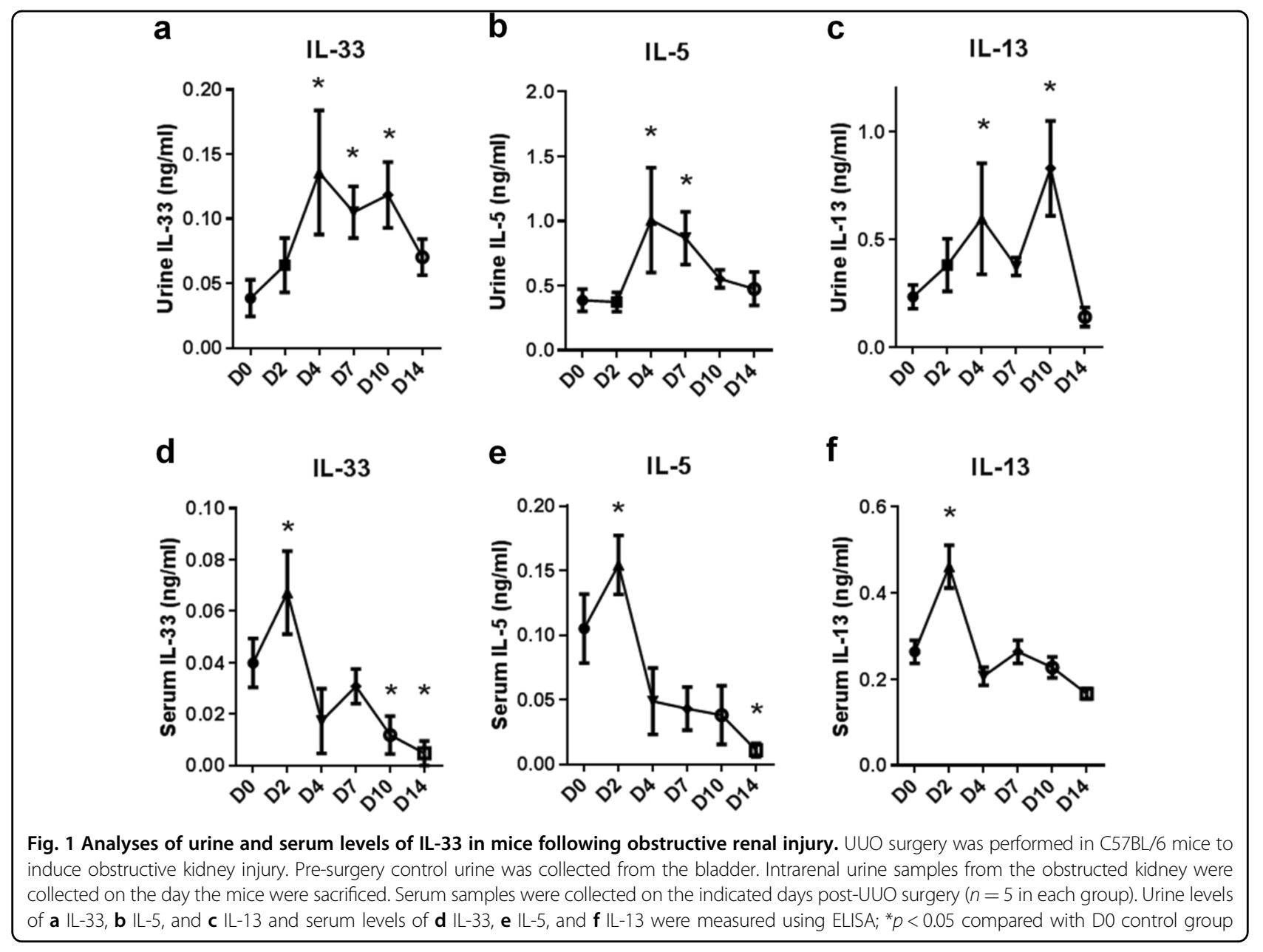

obstructed kidney (Fig. 3b). IL-33+vimentin+ stroma cells were also upregulated in the UPJ in the obstructed kidney (Fig. 3c). No evident IL-33 staining co-localized with Lotus lectin (LTL)-positive proximal tubular cells (Fig. 3d). These results indicate that IL-33 is upregulated in the obstructed kidney and predominantly co-localizes with vimentin + tubulointerstitial cells in the cortex and medullar layers.

\section{UUO upregulated IL-33 expression in KRT5 + hyperplasic urothelium}

In addition to cortical and medullar IL-33 expression in vimentin + cells, we also observed a marked increase in IL-33 expression in the UPJ urothelium (Fig. 3b). In the kidneys of wild-type C57BL/6 mice without renal obstruction, the UPJ urothelium normally consists of a monolayer of keratin 5 (KRT5)-expressing urothelial cells (Fig. 4a, b, areas 1-3). In the UUO kidney, the UPJ urothelium showed marked epithelial cell thickening with an increased number of KRT5+ basal urothelial cells, whereas the KRT5+ urothelium within the fornix remained monolayered (Fig. 4a, b, areas 4-6). UUO induced the proliferation of the monolayer urothelial cells, forming a hyperplasic urothelium with an average of 3-6 layers of KRT5+ basal cells in the UPJ (Fig. 4b). Interestingly, IL-33 expression was only detected and colocalized with multilayered KRT5 + basal cells in the UPJ (Fig. 4b, area 5) and upper urinary track (Fig. 4b, area 6); it did not co-localize with the monolayered KRT5+ medullar urothelial cells within the fornix (Fig. 4b, area 4 and Fig. 4c). Moreover, IL-33 was not detected in uroplakin 3a (UPK3a)-expressing superficial umbrella cells in the UUO kidney (Fig. 4d). These results indicate that IL33 is predominantly expressed in hyperplasic KRT5+ basal cells and that expression of IL-33 is lost during the process of the terminal differentiation of superficial umbrella cells.

\section{Deficiency of IL33 or ST2 attenuated UUO-induced thickening of UPJ urothelium}

Intrarenal hyperplasia is observed in the obstructed kidney ${ }^{17}$. Our results demonstrated that IL-33 was upregulated in the KRT5 + hyperplasic urothelial layer of the UPJ (Fig. 4). We therefore hypothesized that IL-33 may 


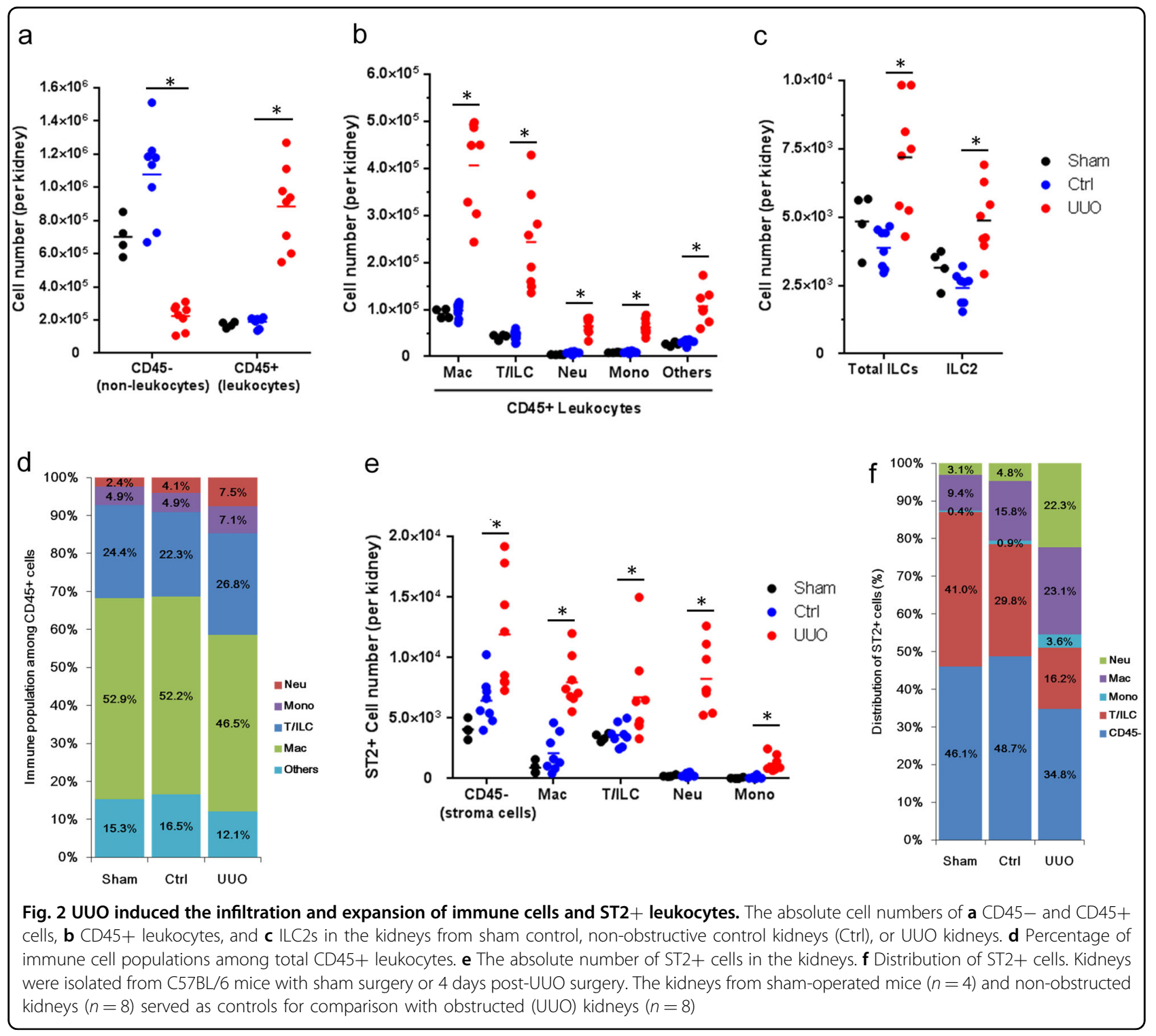

contribute to the hyperplasia of urothelial cells in response to obstructive injury. To test this hypothesis, we compared the levels of urothelial hyperplasia in wild-type C57BL/6, IL33KO, and ST2KO mice following UUO surgery. UUO induced significant urothelial thickening in the UPJ in C57BL/6 mice, whereas deficiency of IL33 or ST2 attenuated UUO-induced UPJ urothelium thickening (Fig. 5a, b). These results indicate that the IL-33/ST2 axis contributes to the hyperplasia of the UPJ urothelium following kidney obstruction.

\section{Deficiency of IL33 altered UUO-induced type 2 cytokine expression}

We next performed qRT-PCR analysis to determine which genes are differentially regulated in the UPJ tissues from wild-type and IL33KO mice following UUO. Two- dimensional PCA was performed to simplify the qRTPCR results (Supplementary Figure S2). PC1 reflected differences in gene expression between UUO UPJ tissues and non-obstructed control tissues from wild-type C57BL/6 mice (i.e., UUO-mediated gene regulation). PC2 reflected differences between IL33KO and C57BL/6 UUO UPJ tissues (i.e., IL-33-dependent gene regulation in UUO UPJ tissues). Upon tissue injury, an increase in IL33 expression activates type 2 immune responses ${ }^{22}$. We found that UUO upregulated the gene expression of type 2 inflammatory cytokines (IL5 and IL13) in UPJ tissues from C57BL/6 mice (Fig. 6a). Deficiency of IL33 attenuated the upregulation of IL5 and IL13 in UPJ tissues. UUO induced the upregulation of amphiregulin (Areg) gene expression, which is derived from activated ILC2s, and this was also attenuated in IL33KO UPJ tissues 
a
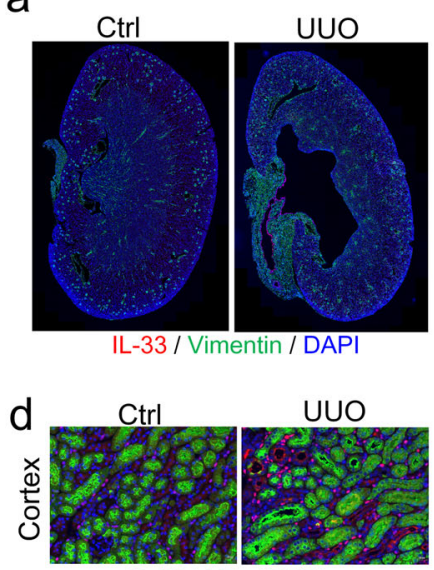

IL-33 / LTL / DAPI b
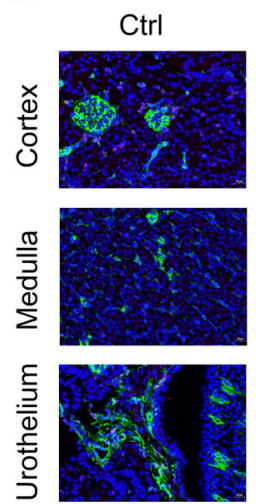

IL-33 / Vimentin / DAPI
C

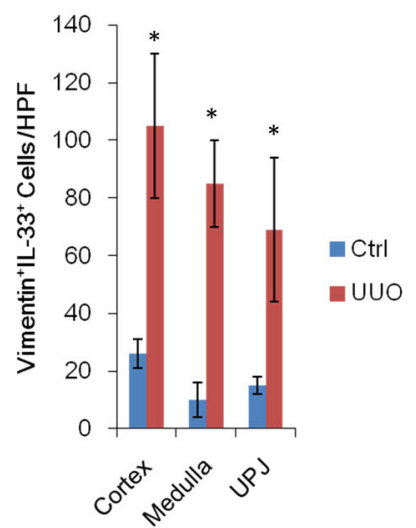

Fig. 3 UUO upregulated IL-33 in interstitial cells in obstructed kidneys. a Immunofluorescence staining of IL-33 (red), vimentin (green), and nuclei with DAPI (blue) in kidney tissues from C57BL/6 mice with UUO surgery. b Expression of IL-33 in vimentin+ cells in the cortex, medulla, and ureter stromal cells. IL-33 was also detected in vimentin-negative multilayered urothelium cells in the UUO kidney. Yellow arrows indicate IL-33 +vimentin+ cells. c Immunofluorescence staining of IL-33 (red), proximal tubules with Lotus lectin (LTL, green), and nuclei with DAPI (blue); ${ }^{*} p<0.05$. d No co-localization of IL-33 with LTL+ cells was detected in the UUO kidney

a

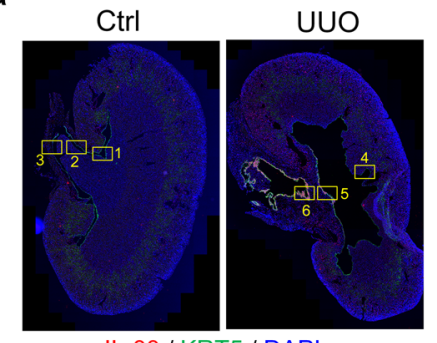

IL-33 / KRT5 / DAPI
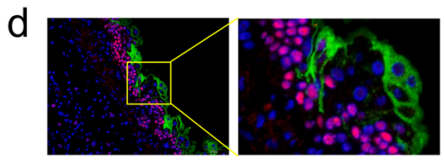

IL-33 / UPK3A DAPI b

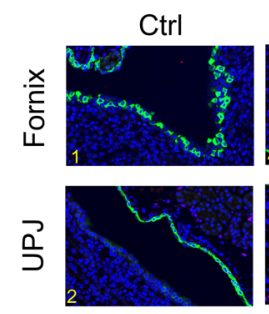

分离

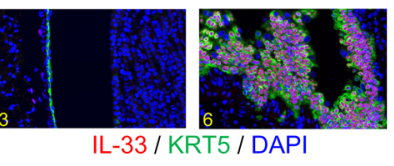

C

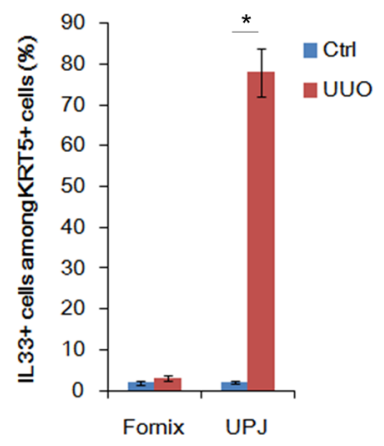

Fig. 4 UUO upregulated IL-33 expression in the hyperplasic intrarenal urothelium. a Immunofluorescence staining of IL-33 (red), KRT5 (green), and nuclei with DAPI (blue) in kidney tissues from C57BL/6 mice with UUO surgery. b Co-localization of IL-33 (red) in KRT5+ (green) urothelial cells in the UPJ but not in the urothelium within the fornix. IL-33 is markedly expressed in the hyperplasic urothelium in the obstructed kidney. $\mathbf{c}$ Quantification of IL-33+ cells among KRT5 cells in the fornix and UPJ of control and UUO kidneys; ${ }^{*} p<0.05$. $\mathbf{d}$ IL-33 is not expressed in UPK3a+ superficial cells

(Fig. 6a). IL4 transcript levels in UPJ tissues did not differ between the IL33KO and C57BL/6 groups.

The protein levels of IL-13 and IL- 5 in hydronephrotic urine samples from C57BL/6 and IL33KO mice were examined to validate the qPCR results. IL33KO mice had lower basal urine levels of IL-13 and IL-5 (Fig. 6b, c). Upon UUO, levels of IL-13 and IL-5 in the hydronephrotic urine samples from IL33KO mice were lower than those in samples from wild-type C57BL/6 mice (Fig. 6b, c). This result indicates that IL-33-mediated type 2 immune responses and ILC2 activation are impaired in IL33KO mice following UUO.

\section{Deficiency of IL33 preserved UUO-induced loss of uroplakins in UPJ tissues}

We next examined whether IL33 deficiency altered the expression of uroplakins and urothelial differentiation markers in UPJ tissues following UUO. The expression of uroplakins in the urothelium layer reflects membrane integrity. Urothelial cells rapidly lose uroplakin expression 


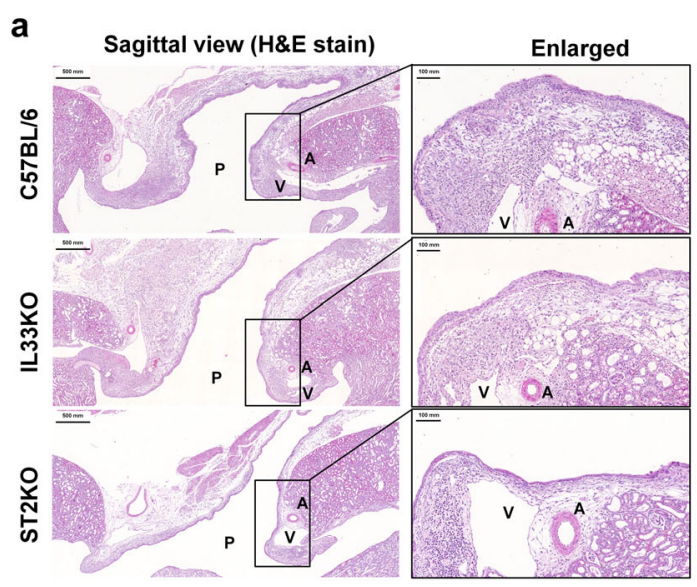

b

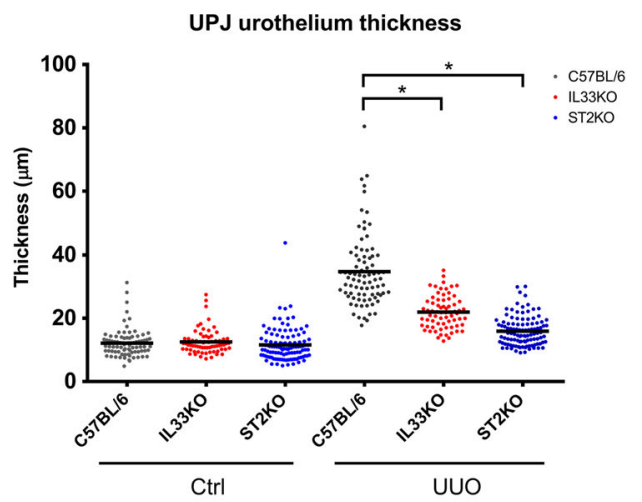

Fig. 5 Deficiency of IL33 or ST2 attenuated UUO-induced hyperplasia of the UPJ urothelium. a Representative images show that deficiency of IL33 or ST2 attenuated UUO-induced intrarenal urothelium hyperplasia. The average UPJ urothelium thickness was determined in wild-type C57BL/6, IL33KO, and ST2KO mice on day 4 after UUO. Fifteen random fields of the urothelium within the UPJ in each mouse were quantified. b Quantification of average urothelium thickness in the non-obstructed control kidneys (Ct) and UUO kidneys from C57BL/6 $(n=8)$, IL33KO ( $n=8)$, and ST2KO mice $(n=8)$. UUO induced significant urothelial thickening in the UPJ in C57BL/6 mice, whereas deficiency of IL33 or ST2 attenuated UUO-induced urothelial thickening; ${ }^{*} p<0.05$

on day 2 post-UUO ${ }^{17}$. We observed that UPK1a, UPK1b, UPK2, UPK3a, and UPK3b were downregulated in UPJ tissues following UUO in C57BL/6 mice (Supplementary Figure S2 and Fig. 6d). Interestingly, deficiency of IL33 preserved the UUO-induced downregulation of the uroplakin genes (Fig. 6d), indicating the preservation or enhancement of urothelial membrane integrity in IL33KO UPJ tissues following UUO.

The protein levels of UPK3a in UPJ tissues from C57BL/ 6 and IL33KO mice were analyzed using western blots (Fig. 6e). We found that the levels of UPK3a protein were reduced in UUO UPJ tissues compared with those in nonobstructed controls. In IL33KO mice, this UUO-induced reduction in the UPK3a protein levels in obstructed UPJ tissues was preserved (Fig. 6e). We next performed immunofluorescent staining to confirm this finding. In UPJ tissues from obstructed kidneys, the expression of UPK3a was lower in the superficial layer of the IL-33expressing hyperplasic urothelium than in the monolayered urothelium in non-obstructed mice (Fig. 6f). The UPJ urothelium from IL33KO mice with UUO showed an intact urothelial layer with preserved UPK3a expression and the lack of IL-33 expression.

\section{Deficiency of IL33 preserved UUO-induced loss of urothelial differentiation signaling}

The increased expression of uroplakins in obstructed UPJ tissues from IL33KO mice may contribute to an increase in urothelial differentiation signaling in the context of IL33 deficiency. Upon injury, urothelial cells begin to proliferate and form a multilayered urothelium ${ }^{23}$. Impaired urothelial differentiation leads to uncontrolled proliferation and metaplasia ${ }^{24}$. Sonic hedgehog ( $\mathrm{SHH}$ ) and FOXA1 have been shown to regulate the terminal differentiation of urothelial cells via BMP4 and $B M P 5^{25-}$ 27. In our model, we found that the urothelial differentiation genes SHH, BMP4, BMP5, and FOXA1 were downregulated by UUO in UPJ tissues from C57BL/6 mice (Fig. 7a). IL33 deficiency prevented the UUOinduced downregulation of urothelial differentiation genes in UPJ tissues from IL33KO mice following UUO (Supplementary Figure S2 and Fig. 7a). Although UUO downregulated $S H H$ gene expression in the UPJ, the protein levels of $\mathrm{SHH}$ in UPJ tissues did not change significantly following UUO in C57BL/6 mice (Fig. 7b). Intriguingly, obstructed UPJ tissues from IL33KO mice showed higher $\mathrm{SHH}$ protein expression compared to those from C57BL/6 mice (Fig. 7b). Immunofluorescent staining also revealed that $\mathrm{SHH}$ protein expression was higher in the obstructed UPJ urothelium layer in IL33KO mice when compared to that in C57BL/6 UUO UPJ tissues (Fig. 7c). Finally, we determined the protein levels of SHH, BMP4, and BMP5 in control and hydronephrotic urine samples and found that IL33 deficiency led to higher levels of SHH, BMP4, and BMP5 in hydronephrotic urine compared with those in urine samples from wild-type C57BL/6 mice (Fig. 7d). These results collectively suggest that deficiency of IL33 is associated with increased urothelial differentiation and the preservation of urothelium integrity (Fig. 7e).

\section{Discussion}

IL-33 has been shown to be involved in various kidney diseases in humans and rodent models ${ }^{6,28-31}$. In cisplatin- 


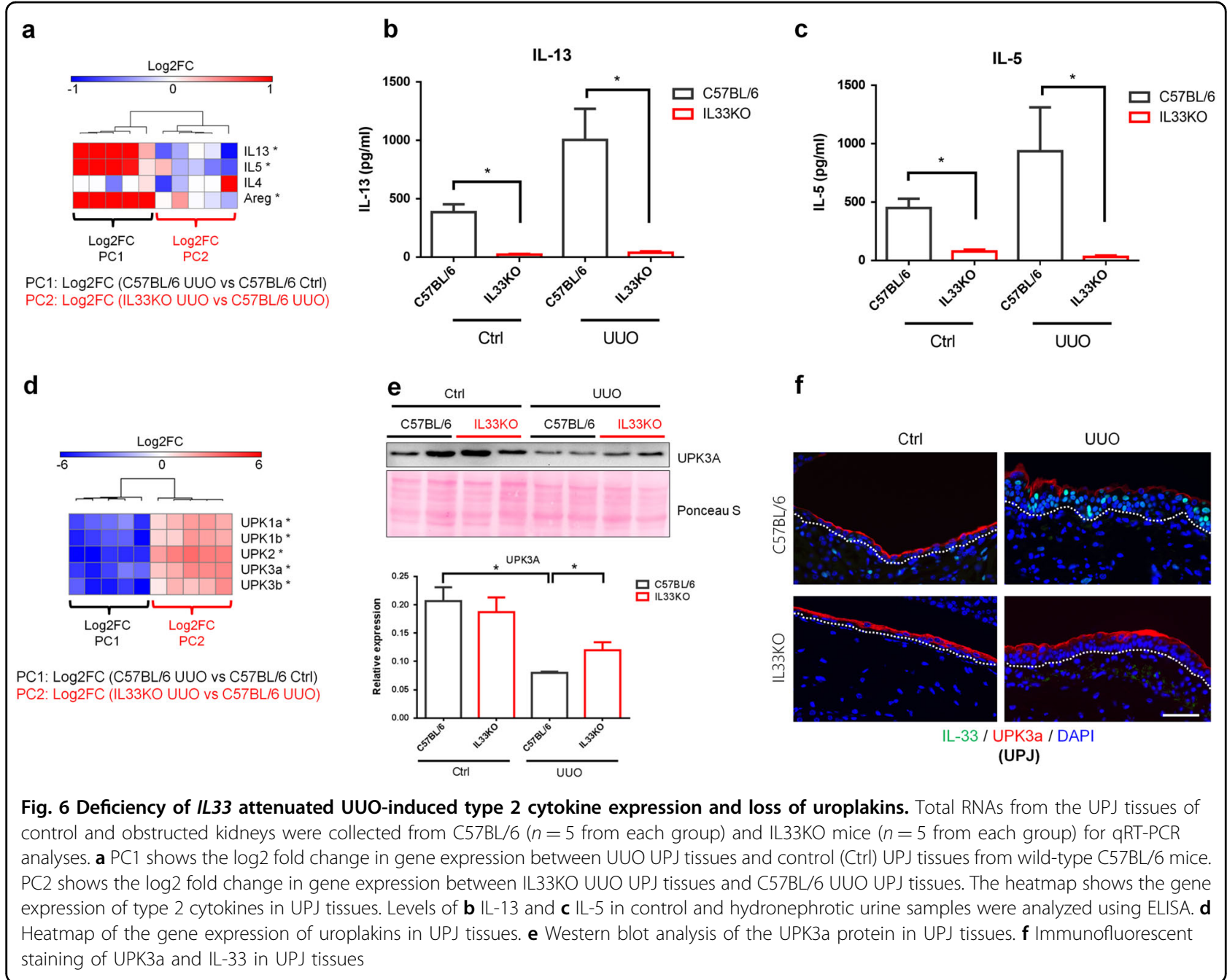

induced acute kidney injury in mice and contrast-induced diabetic nephropathy in rats, serum and kidney IL-33 protein levels are elevated ${ }^{6,32,33}$. Additionally, serum and urine IL-33 levels are increased in patients after renal transplantation $^{34}$. In chronic kidney disease patients, however, IL-33 serum levels are not significantly altered nor correlated with disease severity ${ }^{28}$. Caner et al. demonstrated that serum IL-33 levels in diabetic nephropathy were not associated with kidney injury, although upregulation may be due to diabetes ${ }^{35}$. While it remains controversial whether serum or urine IL-33 levels positively correlate with impaired renal function, the upregulation of tissue IL-33 levels is generally consistent across different renal injury models ${ }^{8,9}$.

In our UUO model, kidney obstruction induced peak serum IL-33 on day 2; levels then returned to their basal values on day 4 and were even lower after day 10 . This suggests that IL-33 likely functions as an acute phase inflammatory cytokine in the UUO model. Persistent elevated IL-33 levels in hydronephrotic urine 4 days post-
UUO suggest that the excretion of IL-33 from the injured kidney is the major source of IL-33 protein in hydronephrotic urine. Interestingly, levels of IL-33, IL-5, and IL13 in hydronephrotic urine returned to basal levels on day 14 post-UUO, suggesting an anti-inflammatory response during the late phase of obstructive nephropathy.

IL-33 has been shown to activate macrophages, neutrophils, ILC2s, eosinophils, and mast cells in various diseases $^{3,36-39}$. In this study, we performed a comprehensive flow cytometry analysis to identify changes in immune cell populations induced by UUO. Among the increased immune infiltrates in the UUO kidney, macrophages and $\mathrm{T}$ cells were predominant. We also surveyed all ST2-expressing cells, which are target cells of IL-33, and determined that macrophages, neutrophils, and $\mathrm{T} /$ ILCs are the major immune targets of IL-33 in the UUO kidney. This observation indicates that IL-33 likely targets multiple immune populations during the acute phase of obstructive nephropathy. Tran et al. reported that IL-33 administration enhanced fungal clearance by increasing 


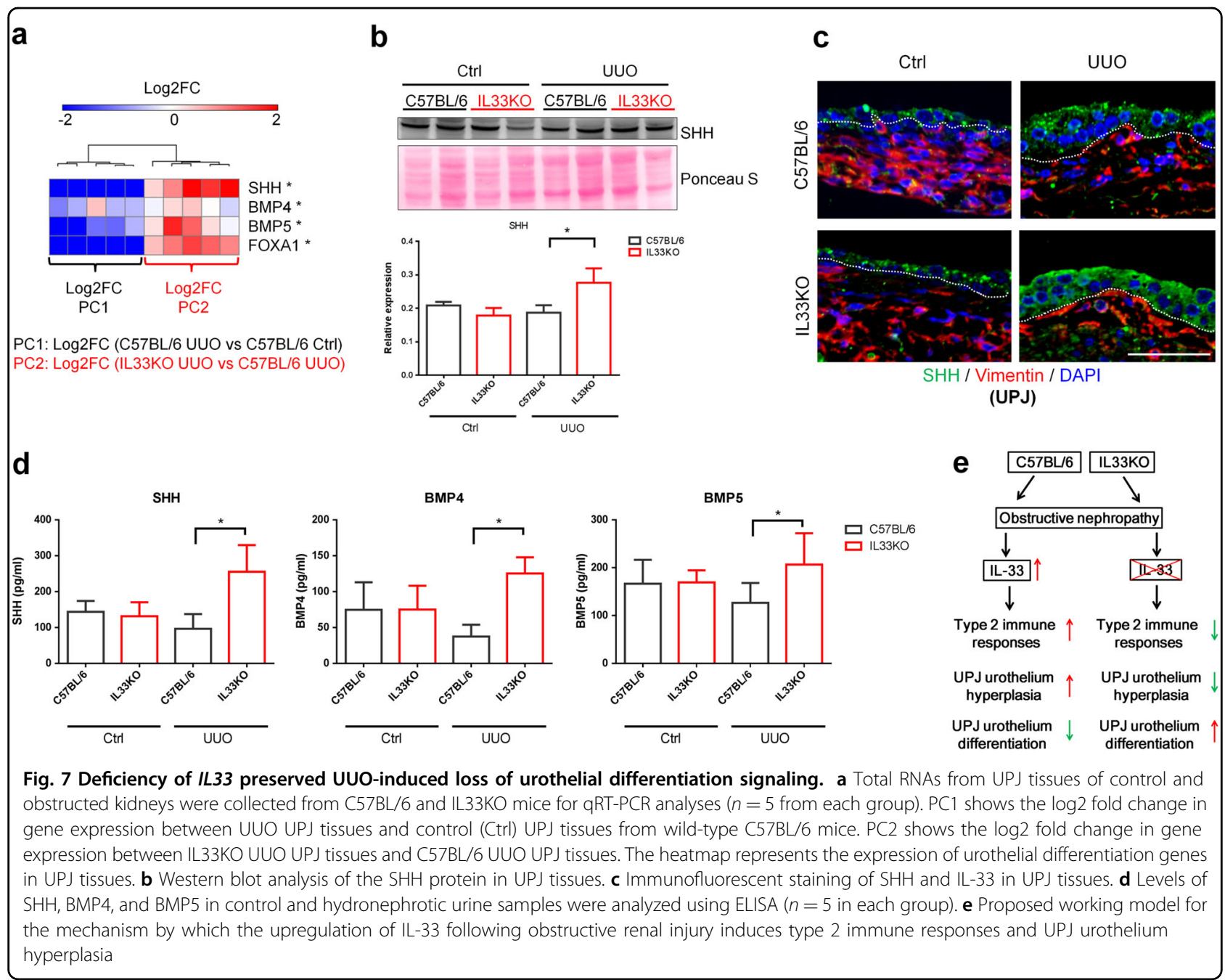

the phagocytic activity of neutrophils ${ }^{40}$. Moreover, deletion of IL13 abolishes the IL-33-mediated polarization of M2 macrophages and renal functional recovery ${ }^{40}$. Whether a specific immune population contributes to the IL33-mediated effector function awaits further investigation.

This study, consistent with our previous findings ${ }^{7}$, showed that IL-33 was upregulated following UUOinduced renal injury. Elevated IL-33 expression was predominantly observed in vimentin + tubulointerstitial cells in the cortex and medullar layers and hyperplasic UPJ urothelium in the UUO kidney, indicating that interstitial myofibroblasts are the major sources of IL-33 in kidney tissues following obstructive injury. Intriguingly, we found that IL-33 expression was also markedly induced in hyperplasic KRT5+ basal urothelial cells in the UPJ but not in the monolayered urothelium of the renal papilla. Deficiency of IL33 or ST2 attenuated UUO-induced UPJ urothelial hyperplasia, indicating a pro-hyperplasic function of the IL-33/ST2 axis in the UPJ urothelium. Girshovich et al. and Vinsonneau et al. showed that ischemia and renal obstruction induced the proliferation of intrarenal urothelial cells via the FGF7-FGFR2 pathway $^{16,17}$. However, transcripts of FGF7 and FGFR2 were not detectable in UPJ tissues from control or UUO kidneys in our model (data not shown), suggesting that IL33-mediated intrarenal hyperplasia is independent of FGF7-FGFR2 signaling.

IL-33 has been shown to be an epithelial barrier alarmin, which elicits an innate response and regulates epithelium integrity upon injury ${ }^{1,41}$. In a cutaneous wound healing model, an IL-33-dependent ILC2 response promotes re-epithelialization and efficient wound closure ${ }^{42}$. A recent study showed that IL-33 induced bronchial epithelial tight junction barrier leakiness via $\mathrm{IL}-13^{43}$. Our study revealed that IL33 deficiency attenuated UUOinduced expression of type 2 cytokines and the loss of uroplakin expression in UPJ tissues, indicating that an IL33 -mediated type 2 immune response likely contributes to the loss of membrane integrity in the UPJ urothelium following kidney obstruction. 
SHH signaling has been shown to inhibit bladder cancer progression by upregulating the stromal production of the urothelial differentiation factors BMP4 and $\mathrm{BMP}^{25}$. Our result demonstrated that IL33 deficiency further upregulated the gene expression of $S H H, B M P 4$, and $B M P 5$ in the obstructed UPJ tissues, which may explain the enhanced urothelial differentiation and uroplakin expression in the UUO UPJ urothelium from IL33KO mice.

Whether IL-33-mediated urothelial hyperplasia is associated with uncontrolled proliferation and urothelial metaplasia awaits further study. Intriguingly, the in vitro treatment of human urothelial cancer cells with IL-33 did not alter cell proliferation (Supplementary Figure S3), suggesting that IL-33-mediated urothelial hyperplasia may occur only under stress conditions such as increased mechanical stretch or changes in urine osmotic pressure during kidney obstruction. Moreover, urothelial IL-33 was only detected in specific regions, particularly the hyperplasic UPJ urothelium, suggesting that the expression of IL-33 is tightly regulated in these cells. Therefore, using bladder or urothelial cancer cells in in vitro models may not be appropriate for studying the biology of IL-33, since the cellular phenotype and culture conditions do not mimic the microenvironment of the obstructed UPJ urothelium. Further study utilizing the primary culture of pelvic or upper urinary track urothelial cells may help to explain how IL-33/ST2 signaling mediates renal injuryassociated UPJ urothelium hyperplasia.

In clinical settings, urothelial hyperplasia is found in children with congenital UPJ obstruction and is associated with type 2 cytokine production ${ }^{19}$. Moreover, UPJ urothelium hyperplasia seems to be common in different kidney injury models ${ }^{16-18}$, and it occurs prior to the development of renal tubulointerstitial fibrosis ${ }^{13,16,17}$. However, it remains unclear whether the degree of UPJ urothelium hyperplasia positively correlates with impaired renal function or kidney fibrosis. This study and our previous work demonstrated that IL33 deficiency resulted in reductions in UUO-induced renal fibrosis ${ }^{7}$, tubular injury, and UPJ urothelium hyperplasia, which may imply that elevated IL-33/ST2 signaling contributes to both pathological outcomes in the renal parenchyma and the UPJ.

Collectively, our study identified a novel and previously unrecognized role of the IL-33/ST2 axis as a molecular sensor to translate increased intrarenal pressure (or stress) into the activation of innate and type 2 immune responses and mediate the hyperplasia of the intrarenal urothelium in obstructive nephropathy by regulating urothelial hyperplasia and differentiation.

\section{Acknowledgements}

This work was supported by Chang Gung Medical Foundation (CMRPG8D0231-3, CMRPG8E0661-3, and CMRPG8G0851 to W.Y.C) and Ministry of Science and Technology, Taiwan (MOST105-2628-B-182A-002-MY3 to W.Y.C, and MOST105-2628-B-001-009-MY3, MOST105-2320-B-001-001 to Y.J.C.) and by the Academia Sinica Career Development Award (104-CDA-L05 to Y.J.C.) in Taiwan. We thank the Center for Research Resources and Development of Kaohsiung Medical University for their technical assistance on TissueFAXS. We also thank the National Laboratory Animal Center (NLAC), NARLabs, Taiwan, for technical support in contract breeding services.

\section{Author details}

${ }^{1}$ Institute for Translational Research in Biomedicine, Kaohsiung Chang Gung Memorial Hospital, Kaohsiung, Taiwan. ${ }^{2}$ Institute of Biomedical Sciences, Academia Sinica, Taipei, Taiwan. ${ }^{3}$ Division of Nephrology, Department of Internal Medicine, Kaohsiung Chang Gung Memorial Hospital and Chang Gung University College of Medicine, Kaohsiung, Taiwan

\section{Conflict of interest}

The authors declare that they have no conflict of interest.

\section{Publisher's note}

Springer Nature remains neutral with regard to jurisdictional claims in published maps and institutional affiliations.

Supplementary information accompanies this paper at https://doi.org/ 10.1038/s12276-018-0047-8.

Received: 5 July 2017 Revised: 20 November 2017 Accepted: 21 December 2017.

Published online: 20 April 2018

\section{References}

1. Martin, N. T. \& Martin, M. U. Interleukin 33 is a guardian of barriers and a local alarmin. Nat. Immunol. 17, 122-131 (2016).

2. Nagarkar, D. R. et al. IL-13 mediates IL-33-dependent mast cell and type 2 innate lymphoid cell effects on bronchial epithelial cells. J. Allergy Clin. Immunol. 136, 202-205 (2015).

3. Yasuda, K. et al. Contribution of IL-33-activated type II innate lymphoid cells to pulmonary eosinophilia in intestinal nematode-infected mice. Proc. Natl Acad. Sci. USA 109, 3451-3456 (2012).

4. Kim, H. Y. et al. Innate lymphoid cells responding to IL-33 mediate airway hyperreactivity independently of adaptive immunity. J. Allergy Clin. Immunol. 129, 216-227 (2012). e211-216.

5. Riedel, J. H. et al. IL-33-mediated expansion of type 2 innate lymphoid cells protects from progressive glomerulosclerosis. J. Am. Soc. Nephrol. 28, 2068-2080 (2017).

6. Akcay, A. et al. IL-33 exacerbates acute kidney injury. J. Am. Soc. Nephrol. 22, 2057-2067 (2011)

7. Chen, W. Y. et al. Upregulation of Interleukin-33 in obstructive renal injury. Biochem. Biophys. Res Commun. 473, 1026-1032 (2016).

8. Chen, W. Y., Li, L. C. \& Yang, J. L. Emerging roles of IL-33/ST2 axis in renal diseases. Int. J. Mol. Sci. 18 (2017). pii E783..

9. Yang, F., Zhu, P., Duan, L., Yang, L. \& Wang, J. IL33 and kidney disease (review). Mol. Med. Rep. 13, 3-8 (2016).

10. Roth, K. S., Koo, H. P., Spottswood, S. E. \& Chan, J. C. Obstructive uropathy: an important cause of chronic renal failure in children. Clin. Pediatr. (Phila.) 41, 309-314 (2002).

11. Klahr, S. \& Morrissey, J. Obstructive nephropathy and renal fibrosis. Am. J. Physiol. Renal Physiol. 283, F861-F875 (2002).

12. Forbes, M. S. et al. Fight-or-flight: murine unilateral ureteral obstruction causes extensive proximal tubular degeneration, collecting duct dilatation, and minimal fibrosis. Am. J. Physiol. Renal Physiol. 303, F120-F129 (2012).

13. Chevalier, R. L., Forbes, M. S. \& Thornhill, B. A. Ureteral obstruction as a model of renal interstitial fibrosis and obstructive nephropathy. Kidney Int $\mathbf{7 5}$, 1145-1152 (2009).

14. Abbott, B. D., Birnbaum, L. S. \& Pratt, R. M. TCDD-induced hyperplasia of the ureteral epithelium produces hydronephrosis in murine fetuses. Teratology $\mathbf{3 5}$, 329-334 (1987)

15. Baumgart, P., Muller, K. M. \& Lison, A. E. Epithelial abnormalities in the renal pelvis in experimental hydronephrosis and pyelonephritis. Pathol. Res. Pract. 176, 185-195 (1983). 
16. Vinsonneau, C. et al. Intrarenal urothelium proliferation: an unexpected early event following ischemic injury. Am. J. Physiol. Renal Physiol. 299, F479-F486 (2010).

17. Girshovich, A. et al. Ureteral obstruction promotes proliferation and differentiation of the renal urothelium into a bladder-like phenotype. Kidney Int. 82, 428-435 (2012)

18. Evan, A. P., Bledsoe, S. B., Smith, S. B. \& Bushinsky, D. A. Calcium oxalate crystal localization and osteopontin immunostaining in genetic hypercalciuric stoneforming rats. Kidney Int. 65, 154-161 (2004).

19. Chiou, Y. Y., Shieh, C. C., Cheng, H. L. \& Tang, M. J. Intrinsic expression of Th2 cytokines in urothelium of congenital ureteropelvic junction obstruction. Kidney Int. 67, 638-646 (2005).

20. Chen, W. Y., Hong, J., Gannon, J., Kakkar, R. \& Lee, R. T. Myocardial pressure overload induces systemic inflammation through endothelial cell IL-33. Proc. Natl Acad. Sci. USA 112, 7249-7254 (2015).

21. Yu, Y. R. et al. A protocol for the comprehensive flow cytometric analysis of immune cells in normal and inflamed murine non-lymphoid tissues. PLOS ONE 11, e0150606 (2016).

22. Hardman, C. \& Ogg, G. Interleukin-33, friend and foe in type-2 immune responses. Curr. Opin. Immunol. 42, 16-24 (2016).

23. Balsara, Z. R. \& Li, X. Sleeping beauty: awakening urothelium from its slumber. Am. J. Physiol. Renal Physiol. 312, F732-F743 (2017).

24. DeGraff, D. J. et al. When urothelial differentiation pathways go wrong: implications for bladder cancer development and progression. Urol. Oncol. 31 802-811 (2013).

25. Shin, $\mathrm{K}$. et al. Hedgehog signaling restrains bladder cancer progression by eliciting stromal production of urothelial differentiation factors. Cancer Cell 26, 521-533 (2014)

26. Yamany, T., Van Batavia, J. \& Mendelsohn, C. Formation and regeneration of the urothelium. Curr. Opin. Organ Transplant. 19, 323-330 (2014).

27. DeGraff, D. J. et al. Loss of the urothelial differentiation marker FOXA1 is associated with high grade, late stage bladder cancer and increased tumor proliferation. PLOS ONE 7, e36669 (2012).

28. Bao, Y. S. et al. Characterization of interleukin-33 and soluble ST2 in serum and their association with disease severity in patients with chronic kidney disease. J. Clin. Immunol. 32, 587-594 (2012).

29. Caporali, A. et al. Soluble ST2 is regulated by p75 neurotrophin receptor and predicts mortality in diabetic patients with critical limb ischemia. Arterioscler. Thromb. Vasc. Biol. 32, e149-e160 (2012).
30. Ryba-Stanislawowska, M., Werner, P., Skrzypkowska, M., Brandt, A. \& Mysliwska, J. IL-33 effect on quantitative changes of CD4+CD25highFOXP3+ regulatory T cells in children with type 1 diabetes. Mediators Inflamm. 2016, 9429760 (2016).

31. Shelite, T. R. et al. IL-33-dependent endothelial activation contributes to apoptosis and renal injury in orientia tsutsugamushi-infected mice. PLoS Negl. Trop. Dis. 10, e0004467 (2016).

32. Onk, D. et al. Melatonin attenuates contrast-induced nephropathy in diabetic rats: the role of interleukin-33 and oxidative stress. Mediators Inflamm. 2016 9050828 (2016).

33. Demirtas, L. et al. The possible role of interleukin-33 as a new player in the pathogenesis of contrast-induced nephropathy in diabetic rats. Ren. Fail. 38, 952-960 (2016).

34. Thierry, A. et al. The alarmin concept applied to human renal transplantation: evidence for a differential implication of HMGB1 and IL-33. PLOS ONE 9, e88742 (2014).

35. Caner, S. et al. Is IL-33 useful to detect early stage of renal failure? Ren. Fail. 36, 78-80 (2014).

36. Monticelli, L. A. et al. Innate lymphoid cells promote lung-tissue homeostasis after infection with influenza virus. Nat. Immunol. 12, 1045-1054 (2011).

37. Chang, Y. J. et al. Innate lymphoid cells mediate influenza-induced airway hyper-reactivity independently of adaptive immunity. Nat. Immunol. 12 631-638 (2011).

38. Iwahana, H. et al. Different promoter usage and multiple transcription initiation sites of the interleukin-1 receptor-related human ST2 gene in UT-7 and TM12 cells. Eur. J. Biochem. 264, 397-406 (1999).

39. De la Fuente, M., MacDonald, T. T. \& Hermoso, M. A. The IL-33/ST2 axis: role in health and disease. Cytokine Growth Factor Rev. 26, 615-623 (2015).

40. Tran, V. G. et al. IL-33 enhances host tolerance to candida albicans kidney infections through induction of IL-13 production by CD4+ T cells. J. Immunol. 194, 4871-4879 (2015).

41. Bruhs, A., Proksch, E., Schwarz, T. \& Schwarz, A. Disruption of the epidermal barrier induces regulatory T cells via IL-33 in mice. J. Invest. Dermatol.16 (2017). pii: S0022-202X(17)33044-0.

42. Rak, G. D. et al. IL-33-dependent group 2 innate lymphoid cells promote cutaneous wound healing. J. Invest. Dermatol. 136, 487-496 (2016).

43. Sugita, K. et al. Type 2 innate lymphoid cells disrupt bronchial epithelial barrier integrity by targeting tight junctions through $\mathrm{IL}-13$ in asthmatic patients. J. Allergy Clin. Immunol. 141, 300-310 (2018). 